\title{
Could adalimumab be used safely and effectively in intestinal Behçet's disease refractory to conventional therapy?
}

\author{
Jihye Park, Jae Hee Cheon \\ Department of Internal Medicine, Institute of Gastroenterology, Yonsei University College of Medicine, Seoul, Korea
}

\section{Article: Long-term safety and efficacy of adalimumab for intestinal Behçet's disease in the open label study following a phase 3 clinical trial (Intest Res 2017;15:395-401)}

Intestinal Behçet's disease (BD), which has pathogenesis and clinical features similar to IBD, tends to occur more prevalently in East Asian countries than Western or Mediterranean regions. ${ }^{1}$ Since intestinal BD is often refractory to conventional treatment such as corticosteroids and immunomodulators, alternative therapies are eventually needed. Currently, anti-tumor necrosis factor $\alpha$ (anti-TNF- $\alpha$ ) agents have greatly improved management of IBD. ${ }^{2}$ Given this evidence, these agents are also under active investigation in the treatment of intestinal BD.

Infliximab is a chimeric monoclonal antibody-based biologic drug that has been reported to be effective for inducing and maintaining remission in patients with intestinal BD. A clinical remission of intestinal lesions and the rapid healing of ulcers after treatment of infliximab was reported in several studies. ${ }^{3}$ Fewer reports have described the use of adalimumab in intestinal BD rather than infliximab. Adalimumab is a completely humanized Ig 1 monoclonal antiTNF- $\alpha$ antibody that binds to TNF- $\alpha$ and prevents it from binding to its receptors. It was the third TNF- $\alpha$ inhibitor, after infliximab and etanercept, to be approved by the U.S. Food and Drug Administration for the treatment of rheumatoid

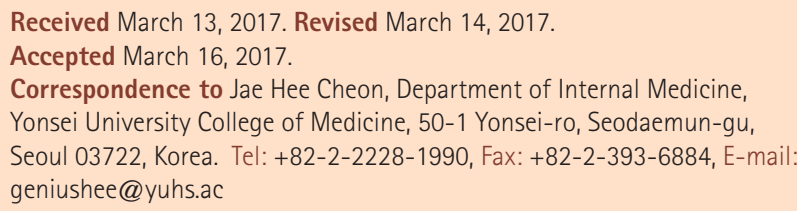

Financial support: None. Conflict of interest: None. arthritis. It has since been approved for further indications including juvenile idiopathic arthritis, psoriatic arthritis, ankylosing spondylitis, CD, UC, hidradenitis suppurativa, psoriasis, and panuveitis. However, it has not been approved for intestinal BD. In Japan, a phase 3, non-randomized, non-controlled, single-arm clinical trial tested adalimumab for intestinal BD refractory to conventional therapy. Of 20 patients, $60 \%$ achieved marked improvement at week 52 and $20 \%$ achieved complete remission at weeks 24 and $52 .{ }^{4}$ Based on this open-label clinical trial and case reports, adalimumab was recently approved and recommended as a standard therapy for intestinal BD in Japan, Taiwan, and South Korea. ${ }^{5,6}$ More evidence should be accumulated for the concrete conclusion that adalimumab is effective in the treatment of intestinal BD.

The study by Inoue et al. ${ }^{7}$ was a follow-up extension study of the aforementioned phase 3 study. The authors found that 100 weeks of long-term adalimumab treatment for patients with intestinal BD who were refractory to conventional therapy was effective without any significant unexpected adverse events. Of the 20 patients enrolled in this study, $60 \%$ of patients at week 52 and $40 \%$ of patients at week 100 achieved marked improvement; $20 \%$ of patients at week 52 and 15\% of patients at week 100 achieved complete remission. The improvement of both clinical and endoscopic disease activities in patients with refractory intestinal BD was observed. The incidence of adverse events during the study was 544.4 events per 100 person-years (190 events) at week 100, which was comparable to 560.4 events per 100 person-years (102

\footnotetext{
๑ Copyright 2017. Korean Association for the Study of Intestinal Diseases. All rights reserved.

This is an Open Access article distributed under the terms of the Creative Commons Attribution Non-Commercial License (http://creativecommons.org/licenses/by-nc/4.0)

which permits unrestricted non-commercial use, distribution, and reproduction in any medium, provided the original work is properly cited.
} 
events) at week 52 . The incidence of serious adverse events was 5.5 events per 100 person-years ( 1 event) and that of severe adverse events was 0 events per 100 person-years at week 52 . Moreover, at week 100, the incidence of serious adverse events was 25.8 events per 100 person-years ( 9 events) and that of severe adverse events was 8.6 events per 100 person-years (3 events). Serious adverse effects after week 52 were considered to be related to intestinal BD itself worsening in these individuals, because 8 serious adverse events with onset after week 52 were observed in 3 patients, and 6 out of 8 events affected the gastrointestinal system. Additionally, Lee et al. ${ }^{3}$ reported that the clinical remission rates after infliximab use at 2, 4, 30, and 54 weeks were $32.1 \%, 28.6 \%$, $46.2 \%$, and $39.1 \%$, respectively in a Korean multicenter study. There was 1 serious infection, but no malignancies or deaths in this study. More recently, an open-label, single-arm, phase 3 study conducted on 18 patients suggested that infliximab induced the healing of main intestinal ulcers in $80 \%$ of patients at week 14. There was 1 intestinal BD patient who suffered serious adverse events, which included worsening of the underlying disease and cataracts. These findings are consistent with those of the adalimumab study conducted by Inoue et al. ${ }^{7}$

There have been several studies on the long-term effects of adalimumab in IBD. In the previous 52-week, randomized controlled study of patients with UC called ULTRA2 (Ulcerative Colitis Long-Term Remission and Maintenance with Adalimumab 2; treatment of moderate to severe UC), 17.3\% of patients showed clinical remission receiving adalimumab and $8.5 \%$ of patients receiving placebo showed remission at week $52 .{ }^{8}$ In the 56-week, randomized controlled study of patients with moderate to severe CD called CHARM (Crohn's trial of the fully Human Antibody Adalimumab for Remission Maintenance), clinical remission rates of $36 \%$ were seen in patients receiving $40 \mathrm{mg}$ of adalimumab every other week, $41 \%$ of patients receiving $40 \mathrm{mg}$ of adalimumab weekly, and $12 \%$ of patients receiving placebo. ${ }^{9}$ In the EXTEND (Extend the Safety and Efficacy of Adalimumab through Endoscopic Healing) trial, $36 \%$ of patients with CD achieved clinical remission at week 52. Furthermore, the 52-week, multi-center, randomized, prospective, open-label trial of patients with CD called DIAMOND (Deep Remission of Immunomodulator and Adalimumab Combination Therapy for Crohn's Disease) reported that the clinical remission rates at 4,26 , and 52 weeks were $77.6 \%, 63.8 \%$, and near $60 \%$, respectively. ${ }^{10}$ Therefore, the effect of adalimumab in intestinal BD might not be significantly different from that in UC patients.

Of note, $20 \%$ of patients at week 52 and $15 \%$ of patients at week 100 achieved complete remission with the use of adalimumab in refractory intestinal $\mathrm{BD} .^{7}$ According to a recent study, the mean percentage of a loss of response to adalimumab in patients with CD was $18.2 \%$, and the annual risk of loss of response was $20.3 \%$ per patient-year. A lack of response to adalimumab developed in $28.5 \%$ to $36 \%$ of patients with UC and loss of response varied between 9 and 18 months. Compared with previous study results, the efficacy of adalimumab in intestinal BD seems to last longer than IBD patients. This possible effect should be further investigated.

Accurate interpretation of this study by Inoue et al. ${ }^{7}$ is limited by the small population and the lack of a control group. Despite this, it provided useful information regarding the long-term outcome of adalimumab in patients with intestinal BD who were refractory to conventional treatments. Adalimumab is a promising treatment option for patients with refractory intestinal BD. However, long-term, largescale, randomized controlled study is yet to be undertaken. Other aspects of the use of TNF- $\alpha$ inhibitors that warrant further exploration include their optimal indications and dosages, as well as whether switching between them should be considered when a lack of adequate response to initial TNF- $\alpha$ inhibitor treatment is observed.

\section{REFERENCES}

1. Kim DH, Cheon JH. Intestinal Behçet's disease: a true inflammatory bowel disease or merely an intestinal complication of systemic vasculitis? Yonsei Med J 2016;57:22-32.

2. Choi CH, Moon W, Kim YS, et al. Second Korean guidelines for the management of ulcerative colitis. Intest Res 2017;15:7-37.

3. Lee JH, Cheon JH, Jeon SW, et al. Efficacy of infliximab in intestinal Behçet's disease: a Korean multicenter retrospective study. Inflamm Bowel Dis 2013;19:1833-1838.

4. Tanida S, Inoue N, Kobayashi K, et al. Adalimumab for the treatment of Japanese patients with intestinal Behçet's disease. Clin Gastroenterol Hepatol 2015;13:940-948.e3.

5. Hisamatsu T, Ueno F, Matsumoto T, et al. The 2nd edition of consensus statements for the diagnosis and management of intestinal Behçet's disease: indication of anti-TNFalpha monoclonal antibodies. J Gastroenterol 2014;49:156-162.

6. Cheon JH. Understanding the complications of anti-tumor necrosis factor therapy in East Asian patients with inflammatory bowel disease. J Gastroenterol Hepatol 2017;32:769-777.

7. Inoue N, Kobayashi K, Maganuma M, et al. Long-term safety and efficacy of adalimumab for intestinal Behçet's disease in the open label study following a phase 3 clinical trial. Intest Res 2017;15:395-401. 
8. Warner B, Harris AW. Adalimumab induces and maintains clinical remission in patients with moderate-to-severe ulcerative colitis. Gastroenterology 2012;143:e42. doi: 10.1053/ j.gastro.2012.03.055.

9. Colombel JF, Schwartz DA, Sandborn WJ, et al. Adalimumab for the treatment of fistulas in patients with Crohn's disease. Gut 2009;58:940-948.
10. Matsumoto T, Motoya S, Watanabe K, et al. Adalimumab monotherapy and a combination with azathioprine for Crohn's disease: a prospective, randomized trial. J Crohns Colitis 2016;10:1259-1266. 